Pacific Journal of Mathematics

HYPONORMAL CONTRACTIONS AND STRONG POWER 


\title{
HYPONORMAL CONTRACTIONS AND STRONG POWER CONVERGENCE
}

\author{
C. R. Putnam
}

Let $T^{*}$ be a hyponormal contraction on a Hilbert space, so that $T T^{*}-T^{*} T=D \geqq 0$ and $\|T\| \leqq 1$. It is shown that if, in addition, $T^{*}$ is completely hyponormal, then the sequence $\left\{T^{n}\right\}_{n=1,2}, \cdots$ converges strongly to 0 as $n \rightarrow \infty$. The result is obtained as a consequence of properties of the solution $w(z)$ of $(T-z I) w(z)=x$, where $x$ is a certain vector in the range of $D$.

1. Let $T$ be a bounded operator on a Hilbert space $\mathfrak{F}$ with spectrum $\sigma(T)$ and point spectrum $\Pi_{0}(T)$. The range and null space of $T$ will be denoted by $R(T)$ and $N(T)$ respectively. If $A$ is any linear manifold in $\mathfrak{S}$, its closure will be denoted by $[A]$. Also, we shall consider the set of numbers $z$ for which $\bar{z} \in \Pi_{0}\left(T^{*}\right)$ and which will be denoted by $\left(\Pi_{0}\left(T^{*}\right)\right)^{*}$.

Let $T_{z}=T-z I$ for any complex number $z$ and let $D$ be a nonnegative self-adjoint operator satisfying

$$
T_{z} T_{z}^{*} \geqq D \geqq 0 \text { for all } z \text { in } C \text {. }
$$

It was shown in Putnam [8] that if $D$ has the spectral resolution

$$
D=\int_{0}^{\infty} u d F_{u}
$$

and if $x$ is any vector satisfying

$$
x=F((s, \infty)) x, \quad s>0,
$$

then $T_{z}^{-1} x$ is bounded and weakly continuous on $C-P$, where $P=$ $\left\{z: z \in \Pi_{0}(T)\right.$ or $\left.\bar{z} \in \Pi_{0}\left(T^{*}\right)\right\}$. (Actually, the set $P$ occurring in [8] was defined differently but should have been defined as above.) This result will be strengthened below to the following

Theorem 1. Suppose (1.1), (1.2) and that $x \in \mathfrak{S}$ satisfies

$$
k_{x} \equiv \int_{+0}^{\infty} u^{-1} d\left\|F_{u} x\right\|^{2}<\infty .
$$

Then there exists a vector-valued function $w(z)$ on $C$ satisfying

$$
T_{z} w(z)=x \text { and }\|w(z)\| \leqq k_{x}^{1 / 2}, \quad z \in \boldsymbol{C},
$$


and having the following properties. At every point $z_{0} \notin \Pi_{0}(T)$, $w(z)$ is weakly continuous, that is, for every $f$ in $\mathfrak{K},(w(z), f)$ is continuous at $z_{0}$. Further, if $\mathfrak{S}$ is separable then, for every $f$ in $\mathfrak{H}$, the function $(w(z), f)$ is Lebesgue planar measurable on the set $\boldsymbol{C}-\left(\Pi_{0}\left(T^{*}\right)\right)^{*}$. In addition, if $\alpha$ is any rectifiable curve in $\boldsymbol{C}$ with arc length measure $m_{\alpha}$ and if $m_{\alpha}\left(\alpha \cap\left(\Pi_{0}\left(T^{*}\right)\right)^{*}=0\right.$ then $(w(z), f)$ is $m_{\alpha}$-measurable as well as $d z(=d x+i d y)$-measurable on $\alpha$.

REMARKs. Note that if $z \notin \Pi_{0}(T)$ then necessarily $w(z)=T_{z}^{-1} x$, and that, for any $f$ in $\mathscr{K},(w(z), f)$ is analytic in $C-\sigma(T)$. Further, it is clear that all vectors $x$ of (1.3) satisfy (1.4) and hence that the set of vectors $x$ satisfying (1.4) is dense in $R(D)$.

That the set $\Pi_{0}\left(T^{*}\right)$ occurring in the statement of Theorem 1 and, more generally, the point spectrum of any bounded operator on a separable Hilbert space, is Lebesgue planar measurable follows from a result of Dixmier and Foiaş [3] as Nikolskaya [7]. We are indebted to K. F. Clancey for informing us of these facts.

Recall that a bounded operator $S$ is said to be hyponormal if $S^{*} S-S S^{*} \geqq 0$ and completely hyponormal if, in addition, there does not exist any non-trivial reducing subspace of $S$ on which its restriction is normal. If $S_{z}=S-z I$, then $S_{z}{ }^{*} S_{z}-S_{z} S_{z}{ }^{*}=S * S-S S^{*}$. Clearly, if $S$ is hyponormal then $\Pi_{0}(S) \subset\left(\Pi_{0}\left(S^{*}\right)\right)^{*}$ and any eigenvector of $S$ belonging to $z$ is also an eigenvector of $S^{*}$ belonging to $\bar{z}$. In particular, $\Pi_{0}(S)$ must be empty whenever $S$ is completely hyponormal. Further, it is easy to see that if $T^{*}$ is hyponormal then (1.1) holds with $D=T T^{*}-T^{*} T$. Consequently, in view of Theorem 1 , we have the following

THEOREM 2. Let $T^{*}$ be completely hyponormal on $\mathfrak{S}$ and let $D=T T^{*}-T^{*} T(\geqq 0)$ have the spectral resolution (1.2). If $x \in \mathfrak{F}$ satisfies (1.4) then there exists a vector-valued function $w(z)$ on $C$ satisfying the conditions of Theorem 1. Thus, relation (1.5) holds and $w(z)$ is weakly continuous at all points $z_{0} \notin \Pi_{0}(T)$. If $\mathfrak{S}$ is separable, then, since $\Pi_{0}\left(T^{*}\right)$ is now empty, $(w(z), f)$ is Lebesgue planar measurable in $\boldsymbol{C}$ and is measurable with respect to arc length measure and to the $d z=d x+i d y$ measure on all rectifiable curves in $\boldsymbol{C}$.

As a consequence of Theorem 2 there will be proved

THEOREM 3. Let $T^{*}$ be completely hyponormal on $\mathfrak{F}$ and suppose that $T$ is a contraction, that is, $\|T\| \leqq 1$. Then $\left\{T^{n}\right\}_{n=1,2, \ldots}$ converges 
strongly to 0 as $n \rightarrow \infty$, that is, $\left\|T^{n} f\right\| \rightarrow 0$ as $n \rightarrow \infty$ for every $f$ in 5 .

REMARKs. It follows from Theorem 3 that if $T^{*}$ is any hyponormal contraction then $T$ can be written as the direct sum $T=$ $T_{1} \oplus N$, where $T_{1}^{*}$ is completely hyponormal, $T_{1}^{n} \rightarrow 0$ strongly as $n \rightarrow \infty$, and $N$ is normal. Clearly, $N=\int z d K_{z}$ can be further decomposed as $N=\int_{|z|<1} z d K_{z}+\int_{|z|=1} z d K_{z}=N_{1} \oplus N_{2}$, where $N_{1}^{n} \rightarrow 0$ strongly as $n \rightarrow \infty$ and $N_{2}$ is unitary. Hence, one has the following

COROLlary 1 of THEOREM 3. Let $T^{*}$ be any hyponormal contraction on a Hilbert space. Then $T=T_{2} \oplus U$ where $T_{2}^{n} \rightarrow 0$ strongly as $n \rightarrow \infty$ and $U$ is unitary, where it is understood that either component of the direct sum may be missing.

Thus, if $T^{*}$ is any completely nonunitary (cf. Sz.-Nagy and Foiaş [11], p. 72) hyponormal contraction, then $T^{n} \rightarrow 0$ strongly as $n \rightarrow \infty$, so that $T$ is of class $C_{0}$. (cf. [11], p. 72). In was shown in [8], p. 167, that if $T^{*}$ is a hyponormal contraction for which $T^{n} \nrightarrow 0$ then $T$ has a nontrivial invariant subspace. The above Corollary yields the stronger result that $T^{*}$ (hence $T$ ) even has a unitary part. Also, it follows from the Corollary that if $T^{*}$ is a hyponormal contraction for which $T^{n} f \nrightarrow 0$ as $n \rightarrow \infty$ whenever $f \neq 0$, then $T$ must be unitary. In case $T^{*}$ is also subnormal, this last result was obtained by Stampfli [10].

COROLlaRY 2 OF THEOREM 3. Let $T$ be a completely hyponormal contraction on a Hilbert space. Then $T^{*}$ is (unitarily equivalent to) the restriction of the adjoint of a unilateral shift to an invariant subspace.

Proof. Actually, every contraction $S$ satisfying $S^{n} \rightarrow 0$ strongly as $n \rightarrow \infty$ is unitarily equivalent to the restriction of the adjoint of a unilateral shift to an invariant subspace (Foiass [4], de Branges and Rovnyak [1, 2]. See also Halmos [5], problem 121, and Sz.-Nagy and Foias [11] p. 95. Note that the unilateral shift in question is, in general, not the simple unilateral shift.

2. Proof of Theorem 1. The proof will be an extension and refinement of the argument given in [8]. Let $z$ be fixed and let $T_{z} T_{z}^{*}$ have the spectral resolution

$$
T_{z} T_{z}^{*}=\int_{0}^{\infty} u d E_{u}^{(z)}
$$


Then, by an argument like that on pp. 165-166 of [8],

$$
\int_{0}^{\infty} \lim _{t \rightarrow 0+}(u+t)^{+1} d\left\|E_{u}^{(z)} x\right\|^{2} \leqq k_{x},
$$

where $k_{x}$ is defined by (1.4). It follows that

$$
E^{(z)}(\{0\}) x=0 \text { and } \int_{+0}^{\infty} u^{-1} d\left\|E_{u}^{(z)} x\right\|^{2} \leqq k_{x},
$$

and hence, for any $z$ in $C$,

$$
y(z)=\int_{+0}^{\infty} u^{-1 / 2} d E_{u}^{(z)} x \text { is defined and }\|y(z)\|^{2} \leqq k_{x} .
$$

Next, let $T_{z}=T-z I$ have the polar factorization (see Kato [6], pp. 334-335)

$$
T_{z}=U(z) G(z),
$$

where $G(z)=\left(T_{z}^{*} T_{z}\right)^{1 / 2}$ and $U(z)$ is partially isometric with initial set $[R(G(z))]$ and final set $\left[R\left(T_{z}\right)\right]$. Then $T_{z} U^{*}(z) y(z)=\left(U(z) G(z) U^{*}(z)\right) y(z)=$ $\left(T_{z} T_{z}^{*}\right)^{1 / 2} y(z)=x$. On putting

$$
w(z)=U^{*}(z) y(z),
$$

one sees that (1.5) follows from (2.3).

Next, it will be shown that the above defined bounded vectorvalued function $w(z)$ on $C$ is weakly continuous at every point $z_{0}$ not in $\Pi_{0}(T)$. It must be shown that $w(z)$ converges weakly to $w\left(z_{0}\right)$, that is, for any $f$ in $\mathfrak{S},(w(z), f) \rightarrow\left(w\left(z_{0}\right), f\right)$ as $z \rightarrow z_{0}$. If this limit relation did not hold however, then, since $w(z)$ is bounded, there would exist a $z_{0}$ and a sequence $\left\{z_{n}\right\}$ such that $w\left(z_{n}\right) \rightarrow p$ (weakly) as $z_{n} \rightarrow z_{0}$ with $p \neq w\left(z_{0}\right)$. It follows from the relation $T_{z} w(z)=x$, on letting $z=z_{n}$ and noting that $\left\|T-T_{n}\right\| \rightarrow 0$, that $T_{z_{0}} p=x$ and, since $T_{z_{0}} w\left(z_{0}\right)=x$, that

$$
T_{z_{0}}\left(p-w\left(z_{0}\right)\right)=0 \text {. }
$$

Since $z_{0} \notin \Pi_{0}(T)$, then $p=w\left(z_{0}\right)$, a contradiction.

There remains then to establish the measurability of $w(z)$ in the sense described in Theorem 1 , at least if $\mathfrak{F}$ is separable. To this end, we first shall show that, whether or not $\mathfrak{S}$ is separable, if $T$ is any operator with the polar factorization of (2.4), then

$$
U(z) \rightarrow U\left(z_{0}\right) \text { strongly as } z \rightarrow z_{0} \text { whenever } z_{0} \notin \Pi_{0}(T) \text {. }
$$

Assume then that $z_{0} \notin \Pi_{0}(T)$. Note (cf. [6], pp. 334-335) that 
$U(z)$ is defined for vectors in $R(G(z))$ by $U(z): G(z) u \rightarrow T_{z} u$ and that $U(z)$ is then extended by continuity to be isometric on $[R(G(z))]$. For $y$ in $N(G(z))\left(=N\left(T_{z}\right)\right), U(z) y=0$. Since $z_{0} \notin \Pi_{0}(T)$, then $N\left(G\left(z_{0}\right)\right)=0$ and so $U\left(z_{0}\right)$ is isometric.

Since $R\left(G\left(z_{0}\right)\right)$ is dense in $\mathfrak{S}$, relation (2.7) will follow if it is shown that

$$
U(z) v \longrightarrow U\left(z_{0}\right) v \text { (strongly) as } z \longrightarrow z_{0} \text {, whenever } v \in R\left(G\left(z_{0}\right)\right) \text {. }
$$

Suppose then that $v \in R\left(G\left(z_{0}\right)\right)$, so that $v=G\left(z_{0}\right) u$ for some vector $u$. In view of $U(z) G(z) u=T_{z} u$ and $U\left(z_{0}\right) G\left(z_{0}\right) u=T_{z_{0}} u$, we have $U(z) v-U\left(z_{0}\right) v=\left(T_{z}-T_{z_{0}}\right) u-U(z)\left(G(z)-G\left(z_{0}\right)\right) u$. Since $\left\|T_{z}-T_{z_{0}}\right\| \rightarrow 0$, hence also $\left\|G(z)-G\left(z_{0}\right)\right\| \rightarrow 0$, as $z \rightarrow z_{0}$, relation (2.8), hence also (2.7), follows. By symmetry, we have also

$$
U^{*}(z) \longrightarrow U^{*}\left(z_{0}\right) \text { strongly as } z \longrightarrow z_{0} \text { whenever } \bar{z}_{0} \notin \Pi_{0}\left(T^{*}\right) \text {. }
$$

Henceforth, it will be supposed that $T$ is the operator occurring in the statement of Theorem 1. By (2.2) and (2.3), $y(z) \in\left[R\left(T_{z} T_{z}^{*}\right)^{1 / 2}\right]=$ $\left[R\left(T_{z}\right)\right]$, and this set is the initial set of $U^{*}(z)$. Since $w(z)=U^{*}(z) y(z)$, it follows that $U(z) w(z)=U(z) U^{*}(z) y(z)=y(z)$. We shall show that

$$
y(z) \longrightarrow y\left(z_{0}\right) \text { weakly as } z \longrightarrow z_{0}, \bar{z}_{0} \notin \Pi_{0}\left(T^{*}\right) \text {. }
$$

If (2.10) did not hold then, since $y(z)$ is (uniformly) bounded in $C$, there would exist a sequence $\left\{z_{n}\right\}$ for which $z_{n} \rightarrow z_{0}$ and $y\left(z_{n}\right) \rightarrow q$ (weakly) as $n \rightarrow \infty$ with $q \neq y\left(z_{0}\right)$. Since $w(z)$ is also bounded, we may choose a subsequence of $\left\{z_{n}\right\}$, which will also be denoted by $\left\{z_{n}\right\}$, such that $w\left(z_{n}\right) \rightarrow p$ (weakly).

Let $f$ be arbitrary in $\mathfrak{S c}$. Then $\left(y\left(z_{n}\right) f\right) \rightarrow(q, f)$ and also $\left(y\left(z_{n}\right), f\right)=\left(U\left(z_{n}\right) w\left(z_{n}\right), f\right)=\left(w\left(z_{n}\right), U^{*}\left(z_{n}\right) f\right)$. In view of (2.9), we have $\left(w\left(z_{n}\right), U^{*}\left(z_{n}\right) f\right) \rightarrow\left(p, U^{*}\left(z_{0}\right) f\right)=\left(U\left(z_{0}\right) p, f\right)$, and hence $q=U\left(z_{0}\right) p$. Since $y\left(z_{0}\right)=U\left(z_{0}\right) w\left(z_{0}\right)$, we see that $q-y\left(z_{0}\right)=U\left(z_{0}\right)\left(p-w\left(z_{0}\right)\right)$. But, as noted earlier, $T_{z_{0}}\left(p-w\left(z_{0}\right)\right)=0$ (cf. (2.6)), so that $p-w\left(z_{0}\right) \in$ $N\left(G\left(z_{0}\right)\right)$ and hence $U\left(z_{0}\right)\left(p-w\left(z_{0}\right)\right)=0$. Thus $q=y\left(z_{0}\right)$, a contradiction, and so $(2.10)$ is proved.

In summary, we see that the vector-valued function $w(z)$ on $C$ is weakly continuous at $z_{0} \notin \Pi_{0}(T)$. The vector-valued function $y(z)$ is weakly continuous at $z_{0}$ if $\bar{z}_{0} \notin \Pi_{0}\left(T^{*}\right)$. Also, the operator-valued function $U^{*}(z)$ on $C$ is strongly continuous and, hence, $U(z)$ is weakly continuous at $z_{0}$ if $\bar{z}_{0} \notin \Pi\left(T^{*}\right)$.

Suppose now that $\mathscr{F}_{\mathcal{C}}$ is separable. Then, as noted earlier, $\Pi_{0}\left(T^{*}\right)$ 
(hence also $\left.\left(\Pi_{0}\left(T^{*}\right)\right)^{*}\right)$ is Lebesgue planar measurable. It will be shown that for any $f$ in $\mathfrak{S}$, the function $(w(z), f)$ is Lebesgue planar measurable on $C-\left(\Pi_{0}\left(T^{*}\right)\right)^{*}$. For, let $\left\{\phi_{n}\right\}(n=1,2, \cdots)$ be any complete orthonormal system for $\mathfrak{F}$. Then $(w(z), f)=(y(z), U(z) f)=$ $\sum_{n=1}^{\infty}\left(y(z), \phi_{n}\right)\left(\phi_{n}, U(z) f\right)$. But each term of the summation is a function continuous at all points $z$ for which $\bar{z}$ is not in $\Pi_{0}\left(T^{*}\right)$. In particular, each such term, and hence the sum, is (planar) measurable on $\left.C-\Pi_{0}\left(T^{*}\right)\right)^{*}$. (The argument is similar to that used in [9], p. 384, in connection with the proof of Stone's theorem on unitary groups.)

Finally, a similar argument establishes the assertion of the last part of Theorem 1 and the proof is now complete.

3. Proof of Theorem 3. Without loss of generality it may be supposed that $\mathfrak{S C}$ is separable. It follows from Theorem 2 that if $w(z)$ is defined by (2.5) and if $f$ is arbitrary in $\mathscr{F}$, then $(w(z), f)$ is (bounded and) measurable with respect to arc length and to the measure $d z=d x+i d y$ on every circle $C_{r}=\{z:|z|=r\}, 0<r<\infty$. Let

$$
y(r)=-(2 \pi i)^{-1} \int_{C_{r}} w(z) d z\left(=-r(2 \pi i)^{-1} \int_{C} w(r t) d t\right),
$$

where $C=C_{1}$ and all circles are oriented positively. It is understood, of course, that $y(r)$ is defined in terms of the relation $(y(r), f)=$ $-(2 \pi i)^{-1} \int_{C_{r}}(w(z), f) d z$ for any $f$ in $\mathscr{E}$ and that the latter integral is a Lebesgue integral. A similar remark applies to the other integrals of this section.

The set $\Pi_{0}(T) \cap\{z:|z|=1\}$ is empty; otherwise, $T$ would have a normal part. (In fact, if $T$ is any contraction and if $z$ is an eigenvalue of $T$ satisfying $|z|=1$ with eigenvector $f$ then $\bar{z}$ is an eigenvalue of $T^{*}$ with the same eigenvector $f$; cf. [11], p. 8.) If $z$ is fixed and $|z|=1$, then, by Theorem $2, w(r z) \rightarrow w(z)$ (weakly) as $r \rightarrow 1-0$. For any fixed $f$ in $\mathfrak{F}$, it follows from (3.1) and the uniform boundedness convergence theorem that

$$
\begin{aligned}
(y(r), f)= & -(2 \pi i)^{-1} \int_{C_{r}}(w(z), f) d z \\
& \longrightarrow-(2 \pi i)^{-1} \int_{C}(w(z), f) d z \text { as } r \longrightarrow 1-0,
\end{aligned}
$$

Thus, $y(r) \rightarrow y(1)$ (weakly) as $r \rightarrow 1-0$. Similarly, $y(r) \rightarrow y(1)$ (weakly) as $r \rightarrow 1+0$. But, if $r>1,-(2 \pi i)^{-1} \int_{C_{r}} T_{z}^{-1} v d z=v$, for arbitrary $v$, so that, if $x$ is any vector satisfying (1.4), $y(r)=x$ for $r>1$ and hence $y(1)=x$. Hence, we have for such vectors $x$, 


$$
y(r) \longrightarrow x \text { (weakly) as } r \longrightarrow 1-0 .
$$

In view of (1.5),

$$
\begin{aligned}
T y(r) & =-(2 \pi i)^{-1} \int_{C_{r}} T w(z) d z \\
& =-(2 \pi i)^{-1} \int_{C_{r}}(T-z+z) w(z) d z, \\
& =-(2 \pi i)^{-1} \int_{C_{r}} z w(z) d z
\end{aligned}
$$

Similarly, one sees that $T^{n} y(r)=-(2 \pi i)^{-1} \int_{C_{r}} z^{n} w(z) d z$ for $n=1,2, \cdots$, and hence

$$
T^{n} y(r) \longrightarrow 0 \text { (strongly) as } n \longrightarrow \infty, \text { for } r<1 .
$$

Next, let $\mathfrak{M}=\left\{v: T^{n} v \rightarrow 0\right.$ (strongly) as $\left.n \rightarrow \infty\right\}$. Since $T$ is a constraction, $\mathfrak{M}$ is a subspace invariant under $T$. Also, by (3.3), each $y(r), r<1$, belongs to $\mathfrak{M}$. Hence, by (3.2), if $u$ is any vector in $\mathfrak{M}^{\perp}, 0=(y(r), u) \rightarrow(x, u)$ as $r \rightarrow 1-0$, and so $x \in \mathfrak{M}$, where $x$ is any vector satisfying (1.4). Since such vectors are dense in $R(D)$, $R(D) \subset \mathfrak{M}$.

Let now $\&$ denote the least subspace containing $R(D)$ and reducing $T$. It will be shown that

$$
\mathfrak{L \subset ⿻}
$$

To see this, note that if $u \in \mathfrak{M}$ then $T u \in \mathfrak{M}$. Also, $T T^{*}-T T^{*}=D$ and hence $T^{n} T^{*} u=T^{n-1} T^{*} T u+T^{n-1} D u$. Since $D u \in \mathbb{M}$, then $T^{n-1} D u \rightarrow 0$ as $n \rightarrow \infty$ and hence $\lim \sup _{n \rightarrow \infty}\left\|T^{n} T^{*} u\right\| \leqq\|T u\|$. Repetition of this argument shows that $\lim \sup _{n \rightarrow \infty}\left\|T^{n} T^{*} u\right\| \leqq\left\|T^{k} u\right\|$ for $k=1,2, \cdots$, and hence $T^{n} T u \rightarrow 0$ as $n \rightarrow \infty$, so that $T^{*} u \in \mathfrak{M}$. Thus, whenever $u$ is in $\mathfrak{M}$ so also are $T u$ and $T^{*} u$. Since $R(D) \subset \mathfrak{M}$, the desired relation (3.4) follows.

It is clear that $\Omega^{\perp}$ also reduces $T$ and that $T \mid \Omega^{\perp}$ is normal. Since $T^{*}$ is completely hyponormal then $\mathbb{2}^{\perp}=0$, and so by (3.4), $\mathfrak{M}=\mathfrak{S}$. This completes the proof of Theorem 3 .

\section{REFERENCES}

1. L. de Branges and J. Rovnyak, The existence of invariant subspaces, Bull. Amer. Math. Soc., 70 (1964), 718-721.

2. Correction to The existence of invariant subspaces, Bull. Amer. Math. Soc., 71 (1965), 396.

3. J. Dixmier and C. Foiaş, Sur le spectre ponctuel d'un opérateur, Colloq. Math. Soc. János. Bolyai (5), Hilbert Space Operators, Tihany (Hungary), (1970), 127-133. 
4. C. Foiass, A remark on the universal model for contractions of G. C. Rota, Com. Acad. R. P. Romîne, 13 (1963), 349-352.

5. P. R. Halmos, A Hilbert Space Problem Book, van Nostrand Co., Princeton, N. J., 1967.

6. T. Kato, Perturbation Theory for Linear Operators, Springer-Verlag, 1966.

7. L. N. Nikolskaya, Structure of the point spectrum of a linear operator; English translation in Math. Notes, 1974, of article in Math. Zametki, 15 (1974), 149-158.

8. C. R. Putnam, Resolvent vectors, invariant subspaces and sets of zero capacity, Math. Ann., 205 (1973), 165-171.

9. F. Riesz and B. Sz.-Nagy, Functional Analysis, Frederick Ungar Pub. Co., 1955.

10. J. G. Stampfli, Spectral subspaces for hyponormal operators, preprint.

11. B. Sz.-Nagy and C. Foiaş, Harmonic Analysis of Operators on Hilbert Space, North Holland-American Elsevier, 1970.

Received November 27, 1974. This work was supported by a National Science Foundation research grant.

Purdue University 


\section{PACIFIC JOURNAL OF MATHEMATICS}

\section{EDITORS}

RICHARD ARENS (Managing Editor)

University of California

Los Angeles, California 90024

\section{J. DugundJI}

Department of Mathematics University of Southern California Los Angeles, California 90007

D. Gilbarg and J. Milgram

Stanford University

Stanford, California 94305
University of Washington Seattle, Washington 98105

\section{ASSOCIATE EDITORS}
E. F. BECKENBACH
B. H. NeumanN
F. WolF
K. YoShIDA

\section{SUPPORTING INSTITUTIONS}

\author{
UNIVERSITY OF SOUTHERN CALIFORNIA \\ STANFORD UNIVERSITY \\ UNIVERSITY OF TOKYO \\ UNIVERSITY OF UTAH \\ WASHINGTON STATE UNIVERSITY \\ UNIVERSITY OF WASHINGTON \\ $\stackrel{*}{*} \stackrel{*}{*} \stackrel{*}{ }{ }^{*}$ AMERICAN MATHEMATICAL SOCIETY
}

The Supporting Institutions listed above contribute to the cost of publication of this Journal, but they are not owners or publishers and have no responsibility for its content or policies.

Mathematical papers intended for publication in the Pacific Journal of Mathematics should be in typed form or offset-reproduced, (not dittoed), double spaced with large margins. Underline Greek letters in red, German in green, and script in blue. The first paragraph or two must be capable of being used separately as a synopsis of the entire paper. Items of the bibliography should not be cited there unless absolutely necessary, in which case they must be identified by author and Journal, rather than by item number. Manuscripts, in triplicate, may be sent to any one of the editors. Please classify according to the scheme of Math. Reviews, Index to Vol. 39. All other communications should be addressed to the managing editor, or Elaine Barth, University of California, Los Angeles, California, 90024.

The Pacific Journal of Mathematics expects the author's institution to pay page charges, and reserves the right to delay publication for nonpayment of charges in case of financial emergency.

100 reprints are provided free for each article, only if page charges have been substantially paid. Additional copies may be obtained at cost in multiples of 50 .

The Pacific Journal of Mathematics is issued monthly as of January 1966. Regular subscription rate: $\$ 72.00$ a year (6 Vols., 12 issues). Special rate: $\$ 36.00$ a year to individual members of supporting institutions.

Subscriptions, orders for back numbers, and changes of address should be sent to Pacific Journal of Mathematics, 103 Highland Boulevard, Berkeley, California, 94708.

\section{PUBLISHED BY PACIFIC JOURNAL OF MATHEMATICS, A NON-PROFIT CORPORATION}

Printed at Kokusai Bunken Insatsusha (International Academic Printing Co., Ltd.), 270, 3-chome Totsuka-cho, Shinjuku-ku, Tokyo 160, Japan.

\section{Copyright (C) 1975 by Pacific Journal of Mathematics} Manufactured and first issued in Japan 


\section{Pacific Journal of Mathematics}

\section{Vol. 57, No. $2 \quad$ February, 1975}

Norman Larrabee Alling, On Cauchy's theorem for real algebraic curves with boundary .......

Daniel D. Anderson, A remark on the lattice of ideals of a Prüfer domain ..................

Dennis Neal Barr and Peter D. Miletta, A necessary and sufficient condition for uniqueness of

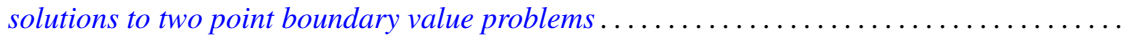

Ladislav Beran, On solvability of generalized orthomodular lattices . . . . . . . . . . ........

L. Carlitz, A three-term relation for some sums related to Dedekind sums . . . . . . . . . .....

Arthur Herbert Copeland, Jr. and Albert Oscar Shar, Images and pre-images of localization

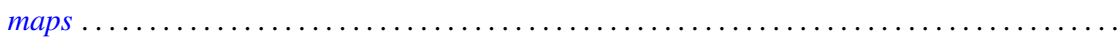

G. G. Dandapat, John L. Hunsucker and Carl Pomerance, Some new results on odd perfect

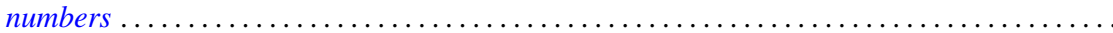

M. Edelstein and L. Keener, Characterizations of infinite-dimensional and nonreflexive

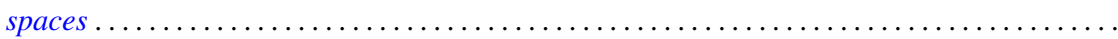

Francis James Flanigan, On Levi factors of derivation algebras and the radical embedding

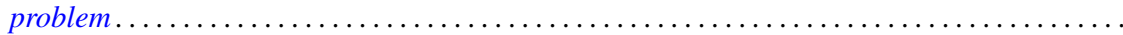

Harvey Friedman, Provable equality in primitive recursive arithmetic with and without

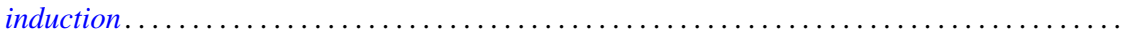

Joseph Braucher Fugate and Lee K. Mohler, The fixed point property for tree-like continua with

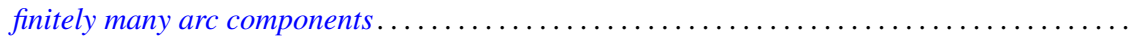

John Norman Ginsburg and Victor Harold Saks, Some applications of ultrafilters in

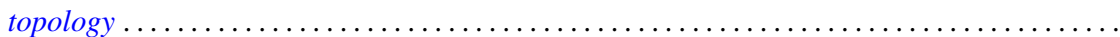

Arjun K. Gupta, Generalisation of a "square" functional equation .....................

Thomas Lee Hayden and Frank Jones Massey, Nonlinear holomorphic semigroups ..........

V. Kannan and Thekkedath Thrivikraman, Lattices of Hausdorff compactifications of a locally

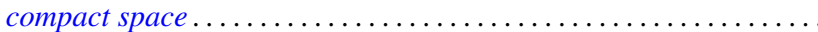

J. E. Kerlin and Wilfred Dennis Pepe, Norm decreasing homomorphisms between group

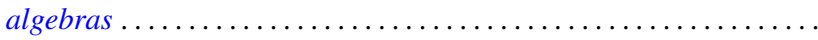

Young K. Kwon, Behavior of $\Phi$-bounded harmonic functions at the Wiener boundary ...

Richard Arthur Levaro, Projective quasi-coherent sheaves of modules .

Chung Lin, Rearranging Fourier transforms on groups...........................

David Lowell Lovelady, An asymptotic analysis of an odd order linear differential equation . . 4475

Jerry Malzan, On groups with a single involution .......................... 481

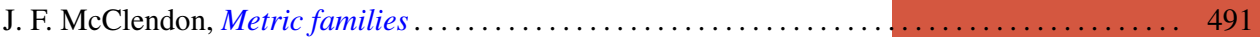

Carl Pomerance, On multiply perfect numbers with a special property .

Mohan S. Putcha and Adil Mohamed Yaqub, Polynomial constraints for finiteness of semisimple rings. .

Calvin R. Putnam, Hyponormal contractions and strong power convergence . . . . . . . . . 531

Douglas Conner Ravenel, Multiplicative operations in $\mathrm{BP} * \mathrm{BP} \ldots \ldots \ldots \ldots \ldots \ldots \ldots \ldots \ldots .539$

Judith Roitman, Attaining the spread at cardinals which are not strong limits . . . . . . . . . 545

Kazuyuki Saitô, Groups of *-automorphisms and invariant maps of von Neumann algebras . . . 553

Brian Kirkwood Schmidt, Homotopy invariance of contravariant functors acting on smooth

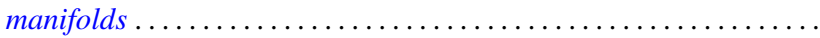

Kenneth Barry Stolarsky, The sum of the distances to $N$ points on a sphere.

Mark Lawrence Teply, Semiprime rings with the singular splitting property.

J. Pelham Thomas, Maximal connected Hausdorff spaces..............

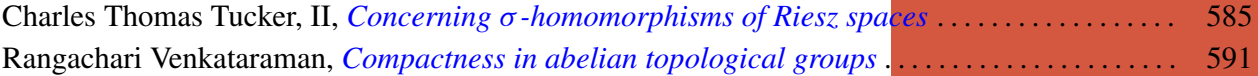

William Charles Waterhouse, Basically bounded functors and flat sheaves . . . . . . . . . . . 597

David Westreich, Bifurcation of operator equations with unbounded linearized part ......... 611

William Robin Zame, Extendibility, boundedness and sequential convergence in spaces of 